Mots. Les langages du politique

$95 \mid 2011$

Sigles et acronymes en politique

\title{
Denis Barbet, Grenelle. Histoire politique d'un mot
}

\section{Éléonore Yasri-Labrique}

\section{(2) OpenEdition}

\section{Journals}

Édition électronique

URL : https://journals.openedition.org/mots/20159

DOI : $10.4000 /$ mots.20159

ISSN : 1960-6001

\section{Éditeur}

ENS Éditions

\section{Édition imprimée}

Date de publication : 1 mars 2011

ISBN : 978-2-84788-307-7

ISSN : 0243-6450

\section{Référence électronique}

Éléonore Yasri-Labrique, «Denis Barbet, Grenelle. Histoire politique d'un mot », Mots. Les langages du politique [En ligne], 95 | 2011, mis en ligne le 01 mars 2013, consulté le 23 avril 2022. URL : http:// journals.openedition.org/mots/20159; DOI : https://doi.org/10.4000/mots.20159 


\title{
Compte rendu de lecture
}

\author{
Grenelle. Histoire politique d'un mot \\ Denis Barbet \\ 2009, Rennes, Presses universitaires de Rennes (Res publica), 280 pages
}

Avec son ouvrage intitulé Grenelle. Histoire politique d'un mot, Denis Barbet, maître de conférences en science politique à l'IEP de Lyon, nous propose un voyage tridimensionnel, historique, sociopolitique et lexical dans l'univers d'un mot devenu banal mais dont le parcours signifiant est à la fois complexe et crucial. Dès que l'on s'empare du livre, une question se pose : peut-on (et derrière ce «on» se cachent par exemple les jeunes générations qui, en quête d'information via Internet, se trouvent quotidiennement confrontées à des formules répondant davantage à ce que Patrick Charaudeau nomme «l'impératif de captation »), peut-on donc véritablement saisir dans leur totalité les significations et les implications de ce nom propre en passe de se transformer en nom commun, sans en connaître à la fois les origines et les résonances dans l'histoire politique française depuis plus de quarante ans? En effet, cette expression, apparue en mai 1968, appartient d'abord au vocabulaire de cette période-là, comme le souligne Maurice Tournier lorsqu'il écrit : «Ce nom propre, devenu label commun [...] localise quai de Grenelle à Paris le lieu des pourparlers qui conduisent au "Projet de protocole d'accord" des 25-27 mai 1968 [et] marque le point culminant des événements» (Les mots de mai 68, 2007, p. 62). Elle connaît une nouvelle fortune en $\mathbf{2 0 0 7}$, année de l'élection de Nicolas Sarkozy à la présidence de la République, avec la revendication d'un «Grenelle de l'écologie» puis la mise en place du «Grenelle de l'environnement». Quels sont les enjeux de l'utilisation récurrente de ce mot? À quoi fait-elle écho sur le plan social? Quelles sont les attentes, les réactions et les interactions créées par cette réalité langagière amplement médiatisée? C'est à ces questionnements, entre autres, que s'attelle le travail de recherche, dense et rigoureux, mené par Denis Barbet, progressant avec clarté entre préoccupations sociohistoriques, analyses de discours (notamment politiques et médiatiques) et réflexions d'ordre lexicologique.

L’ouvrage est divisé en six grands chapitres, eux-mêmes composés de plusieurs paragraphes organisés de manière chronologique ou thématique - selon la teneur plus ou moins historique, plus ou moins sociologique ou linguistique, du contenu - et agrémentés d'encadrés permettant une synthèse des 
principaux points de repère. L'introduction est précédée d'une table des sigles, facilitatrice de la lecture et de la compréhension; la conclusion est suivie d'annexes qui présentent en particulier des tableaux et des textes représentatifs de l'interdiscours construit autour du mot Grenelle. Le chapitre 1, qui a pour titre "À la recherche des Grenelle : avant, pendant et après 2007 ", ancre la première moitié de l'ouvrage dans une perspective historique non pas centrée autour de la naissance du terme en politique fin mai 1968 mais sur sa réappropriation trente-neuf ans plus tard par les différents acteurs sociaux concernés de façon prioritaire par la protection de l'environnement. Denis Barbet explique toutefois dans son introduction, faisant écho aux propos de Maurice Tournier, que :

Dans le cas présent donc, c'est un nom de lieu (la rue de Grenelle, qui reliait Paris au village de Grenelle) qui est d'abord devenu un nom d'événement par dérive métonymique. Le mot réfère en effet aux négociations qui se sont déroulées du 25 au 27 mai 1968, entre les représentants des syndicats, du patronat et du gouvernement, dans la salle des commissions de l'ancien hôtel du Châtelet, affecté au ministère du Travail depuis 1905, situé 127, rue de Grenelle. (p.12)

Il précise un peu plus loin que cette table ronde peut être considérée comme le «référent originel» ou le «porteur initial» du terme Grenelle et que les autres événements ainsi désignés peuvent être appelés « référent cible» ou « référent discursif» (p.13). C'est en tout cas du référent cible «Grenelle de l'environnement» qu'il est longuement question dans les chapitres 2 et 3, l'un se penchant sur «l'invention» de l'énoncé et du concept qu'il recouvre, l'autre s'attachant à en explorer «les enjeux politiques et institutionnels». La seconde moitié du livre, sans occulter les aspects historiques et sociopolitiques liés à cette dénomination de plus en plus couramment employée pour désigner une grande réunion multipartite, est davantage consacrée à une exploration sociolinguistique de ce toponyme devenu, par antonomase avec fonctionnement synecdotique, l'hyperonyme de «négociation au sommet». Le chapitre 4 porte sur «l'évolution des usages de Grenelle» entre 1987 et 2007 tandis que le suivant s'intéresse au passage «du référent initial au référent discursif». Enfin, le dernier chapitre, «la recette du Grenelle à la française», correspond à une tentative, comme l'indique l'auteur lui-même, « de dégager une logique sémantique dans l'usage de ce nom propre devenu plutôt commun, en soulignant des convergences entre les expériences historiques et entre des usages linguistiques, qui contribuent à la construction progressive du sens social actuel du mot Grenelle» (p.19).

Au cœur de cette étude se trouve donc un procédé antonomasique qui dépasse largement le simple fait d'utiliser un nom propre comme un nom commun et qui, de plus, s'inscrit dans la durée. Dès le début de son enquête, Denis Barbet signale avec conviction que :

La figure du Grenelle est loin d'être nouvelle. Elle n'est donc pas réductible à un phénomène de mode langagière, malgré des effets indéniables d'imitation, sur lesquels nous reviendrons. Bien qu'il se généralise et s'accélère en 2007, sous la pression de 
l'actualité et d'une couverture médiatique sans précédent, le processus d'antonomase était déjà engagé, sinon le terme n'aurait sans doute pas été (ré)activé par les «inventeurs» ou promoteurs du Grenelle de l'environnement en particulier. (p. 21)

L'auteur recense près de dix Grenelle de 1983 à 2005, impulsés par des membres issus des différents gouvernements de la France, dont certains semblent avoir été gommés de la mémoire collective, mais qui néanmoins ont marqué l'histoire politique et la matérialité discursive françaises. Il explique également que ces Grenelle sont une coproduction de l'État et de la «société civile» (p.39) et, qu'à travers l'emploi de cette expression, ces grandes tables rondes ont eu un retentissement d'autant plus large dans la vie sociale et politique du pays : «Le pouvoir ou celui qui vise à l'exercer montre ainsi qu'il accède aux «aspirations de la société [...] II plaide ainsi une cause partagée, sans que lui soit apportée une contradiction.» (p.41) Plus qu'une manifestation de la démocratie, les Grenelle sont avant tout des idées et des mots qui participent de la rhétorique politicomédiatique française. Avec cette formule, on plonge dans les arcanes d'une argumentation basée sur la notion de rassemblement et qui correspondrait à ce que Christian Plantin, se référant aux théories prenant pour norme le vrai, résume de la sorte : «Le but de l'activité argumentative est la construction d'un consensus, la résolution des différences d'opinion.» (L'argumentation, 1996, p. 19) En ce qui concerne plus particulièrement le «Grenelle de l'environnement», Denis Barbet nous propose une grille de lecture mettant en perspective les étapes de cette construction à la fois politique et médiatique qui occupe toute l'année 2007 ainsi que les acteurs en présence : Nicolas Sarkozy d'abord en campagne puis président de la République, les autres candidats, les leaders écologistes dont l'énigmatique Nicolas Hulot, les ministres concernés, mais également les ONG et les syndicats, sans oublier les citoyens - censés être associés aux décisions à travers diverses « procédures de concertation » (p. 88-89) - et les experts, professionnels ou scientifiques dont le statut reste flou, les signifiés du terme expertise étant eux-mêmes sujets à fluctuation tout au long de ces négociations.

Le travail du politologue, confronté aux précisions historiques et aux interprétations des événements ainsi retracés, rejoint donc constamment celui du lexicologue, curieux de recenser et de comprendre les évolutions dans l'usage des mots, concomitamment vecteurs et miroirs de l'expérience sociale. L'auteur choisit alors de se concentrer sur la sémantique du nom Grenelle tout au long de la deuxième moitié du livre, en prenant pour référence première le texte intégral du journal Le Monde de 1987 à 2007. Dès le début du chapitre 4, il précise qu'il distingue «quatre usages possibles du mot Grenelle, soit comme : antonomase [...], métonymie [...], toponyme, désignant un simple lieu [...], référence à mai 1968 [...] » (p.135). Il en explore la distribution générale puis s'attaque à une analyse discursive détaillée à travers une réflexion approfondie sur les différentes modalités d'antonomase incluant notamment l'étude du cotexte, des déterminants utilisés, des formes de la complémentation, de la 
présence ou non des guillemets, de l'apparition de dérivés et composés lexicaux. Il conclut alors qu'il s'agit d' « un processus antonomasique inachevé », position qu'il justifie de la sorte :

La figure n'est pas pleinement lexicalisée. Bien sûr, l'établissement d'un sens lexical, i.e. l'entrée dans le vocabulaire commun, constitue le véritable critère de lexicalisation, mais la littérature retient en principe trois critères de lexicalisation, ni absolus, ni irréversibles [...] : (i) la lexicographisation, qui se traduit par l'entrée dans les dictionnaires de noms communs et la langue; (ii) la marque de l'accord au pluriel (Grenelles) et (iii) la perte de majuscule (grenelle). Force est de constater que ces conditions sont encore incomplètement ou du moins inégalement satisfaites. (p. 148)

Denis Barbet s'intéresse par la suite aux «différences entre les Grenelle tentés ou organisés» (p.154), cherchant à mieux cerner les glissements de sens entre le référent initial et les multiples référents discursifs ainsi que les subtilités des mots qui pourraient être proches ou concurrents, tels que processus ou encore table ronde, débat, négociation, sommet, assises, réunion, consultation (p.168169). Il en étudie les connotations et les ramifications, sans négliger les représentations liées au terme Grenelle en France et à l'étranger. Le passage consacré à la réception de ce mot par le grand public (fin du chapitre 5) est d'ailleurs significatif des tensions intracommunautaires qui peuvent se cristalliser autour de certains phénomènes langagiers. Le livre s'achève néanmoins sur une métaphore culinaire qui signale au lecteur que «Grenelle fait [...] recette» (p. 184) aussi bien dans les discours officiels que les interactions sociales, par exemple au niveau des partis politiques ou des associations citoyennes, avec des nuances, des affinements, des particularités... Denis Barbet estime que :

Lesté par d'autres expériences intermédiaires, dans un contexte politique, administratif et idéologique spécifique, le Grenelle de 2007 «empile» de nouveaux traits sémantiques, qui actualisent la forme et les contours de la négociation, apparaissant comme autant de «nouvelles» exigences sociales. (p. 221)

Il finit par s'interroger sur ce Grenelle «enrichi » et sur cette «classe » d'expériences et d'apports sémantiques nouveaux, tirant un premier bilan multifocal des événements et des verbalisations qui ont fait en 2007 le «Grenelle de l'environnement», en s'appuyant notamment avec profit sur les ressources disponibles sur Internet. D'un côté, il envisage un «scénario plutôt favorable à la pérennisation de l'expression » (p. 240); de l'autre, il note qu'il existe une "overdose déclarée de Grenelle [qui] témoigne que la propagation de la formule a dépassé pour certains le seuil de saturation » (p. 241). L'aventure de ce mot continue donc, irrégulière, imprévisible, passionnante, ballottée par les flots de l'Histoire et de la langue, ineffablement portée par la conscience collective.

Éléonore Yasri-Labrique

ARSER-DIPRALANG/EA 739, Montpellier 3

yeleonore_2000@yahoo.fr 